\title{
Discussion on University Laboratory Safety Management under the New Situation
}

\author{
Hanguang Lang ${ }^{1}$, Haifeng Yang ${ }^{2, *}$ \\ ${ }^{1}$ Logistics Security Office, Harbin Institute of Technology (Weihai), Weihai, Shandong, 264209, China \\ ${ }^{2}$ School of Materials Science and Engineering, Harbin Institute of Technology (Weihai), Weihai, Shandong, \\ 264209, China \\ *Corresponding author
}

Keywords: Institution of higher education; laboratory safety; laboratory management.

\begin{abstract}
: laboratory is the important practice base for colleges and universities to cultivate innovative practice talents. Laboratory safety is a key factor to determine whether laboratory teaching and research work can be carried out smoothly. The level of laboratory safety management to a certain extent reflects the comprehensive management level and personnel cultivation quality. This paper elaborates the current situation and existing problems of university laboratory experiment safety management under the new situation, and proposes some specific implementation suggestions for reference to improve safety management from perspectives of strengthening laboratory safety infrastructure, carrying out laboratory safety education for teachers and students, and formulating feasible laboratory safety management system.
\end{abstract}

\section{Introduction}

Laboratory is the important base for talent cultivation and scientific research in universities. Laboratory safety is the basic guarantee for normal laboratory work to be carried out [1-3]. University laboratory safety construction and management as an important component of laboratory construction and management is also the precondition for other work concerning each laboratory manager and participant, as well as an important element of safe and stable campus construction.

After entering the 21st century, with the continuously developing higher education, expanding school-running scale and the increasing school-running level, the types and quantities of university laboratories are also increasing, the scale and function of laboratories are constantly improving, with safety accidents occurred occasionally, which increases the difficulty of laboratory safety management. So, it is urgent to improve university laboratory safety management level $[4,5]$.

\section{Types and causes of university laboratory safety accidents}

University laboratory safety management accidents are reported from time to time. After combining the types and damage level of laboratory safety management safety accidents in recent years, common laboratory safety management accidents can be roughly divided into four categories: fire, explosion, corrosion, poisoning, electromechanical injury and equipment injury. The causes of various laboratory safety accidents are summarized as follows:

Fire: In the university laboratories, most fires involve electricity use. In response to various scientific research tasks, teachers and students pull the wires randomly, and connect wires seriously. They do not pay attention to wire inspection and maintenance. Most wires are aging more, which are easy to cause fire accidents. Most fire accidents are caused by improper use or power management, inappropriate operation, and wire aging, etc.

Explosion: University laboratory explosive accidents often occur in laboratories with inflammable and explosive materials or pressure vessels, usually caused by the storage conditions not up to requirements, or non-standard high-pressure vessel reconditioning; 
Corrosive poisoning: laboratories storing chemicals and highly toxic substances are prone to toxic accidents, which are mostly caused due to improper operation.

Electromechanical injury: are accidents are prone to occur in mechanical laboratories with high-speed revolving or impacting motion, which are mainly caused by violation operation, equipment aging and ill management.

\section{Current situation of university laboratory safety management}

Under the current condition, despite of a lot of manpower and material resources invested in the safety management of laboratories, and laboratory safety management greatly improved, it is urgent to improve the safety hardware construction in laboratory, strengthen teachers and students" safety awareness, and feasible laboratory safety management system guarantee. Compared with safety management work in foreign universities, there still exists big gap [6].

\subsection{Defective laboratory safety basic condition configuration}

Since reform and opening-up, with the expanding school-running scale and increasing volume of students, there are higher requirements for the safety management of university laboratories and higher requirements for the quality and quantity of some basic laboratory safety facilities. But due to long-term limitation of lacking fund investment and lack of security awareness, some basic security facilities of university laboratories such as fire extinguishers, smoke alarms, anti-virus, anti-radiation, ventilation and dust removal cannot be satisfied, let alone, laboratory waste recycling, harmless treatment equipment and necessary personnel first-aid facilities and drugs.

\subsection{Single safety education and poor security awareness}

Currently, safety education carried out by Chinese universities is limited to the fire protection, regular inspections, and public security experience implemented by school security department. The single and boring safety education organization cannot attract frontline managers and laboratory users" interest. Meanwhile, the university leadership and grassroots, ideas of emphasizing teaching and research, ignoring safety and environmental protection exist in university leadership and basic level so that laboratory safety management awareness cannot be deeply penetrated in people's minds. Poor safety awareness and low level of laboratory safety management lead to frequent chemical burns, poison gas poisoning, biological infections and other laboratory safety accidents during teaching and scientific research process.

\subsection{Dull laboratory management system and difficulty in guaranteeing implementation}

Laboratory safety management involves a lot of problems, mainly covering fire and explosion accidents, biological and toxic accidents, electromechanical injury accidents, and safe waste treatment. It requires feasible and sound laboratory safety management system to avoid laboratory safety accidents. Stable system implementation is an important guarantee for safety management. However, laboratory safety management system currently carried out in universities are regulations formulated several years or even a decade ago, empty and unsound, without standardization. They can no longer be used for laboratory safety management under the new situation. Any work development must be guaranteed by excellent system execution. Improving system implementation is the inevitable requirement for laboratory safety management development and perfection. Without system execution, there will be no competitiveness and no developmental force. Only with strong execution ability can the laboratory safety management system be executed steadily. Current laboratory management system is hard to be implemented, leading to long-term stagnant laboratory safety management. 


\section{Reform measures of university laboratory management under the new situation}

University laboratory safety management is an important and indispensable component of laboratory construction and management. Laboratory safety management as an important task for universities during the laboratory planning and design from the beginning to reconstruction and expansion until the operation and maintenance. Colleges and universities should strengthen safety loophole investigation in every step of the laboratory construction process, and focus on eliminating laboratory safety loopholes. Meanwhile, schools should make systematic design and plan for laboratory safety work, establish and improve laboratory safety management system, strengthen safety education for teachers and students, carry out research and study in safety domain, set up long-term mechanisms through institutionalization, standardization and normalization so as to guarantee the sustainable and safe laboratory management.

\subsection{Continuous improvement of laboratory safety hardware conditions}

During laboratory safety management, hardware conditions involving safety management are important basis to achieve high level the laboratory management. The laboratory safety condition construction and improvement should be carried out sync with laboratory construction. The laboratory should be equipped with sufficient universal safety facilities such as extinguishers, fire-fighting sand and other fire-fighting equipment. The safe passage should be set in the corridor. Specialized safety hardware should be equipped in various professional laboratories such as ventilation ducts, welding masks, and welding gloves in the welding laboratory, fume hood, eye wash, gas mask in the chemical laboratory, waste medium, high temperature and high pressure disinfection device for uniform treatment in a biological laboratory, video surveillance, anti-theft alarm system installed in the experimental material depot.

Continuously improving security hardware conditions in university laboratories can provide powerful support for laboratory safety management improvement. With the improved awareness and investment of the country and universities in laboratory safety management, the laboratory safety hardware basis will be greatly enhanced.

\subsection{Extensive implementation of safety education and improvement of teachers and students' safety awareness}

The principal part of security is people, as well as the main cause of safety problems and the recipients of security accidents. Tracing the source, people's safety awareness is the most important. It is inevitable to be exposed to chemicals when entering in laboratory. Many chemicals can damage human body. The most common is the laboratory acid and alkali. Concentrated acid and alkali will burn the skin and cause serious results if splashed into the eyes. It requires laboratory personnel to know the nature of chemicals very well, master the methods and safeguard procedures, and develop good experimental habits to lower safety accident occurrence.

Conducting systematic safety publicity education is a good way for sound teaching laboratory safety work. In accordance with "all personnel, full course, comprehensive" requirements, it is necessary to carry out systematic learning of laboratory safety knowledge and continuously improve teachers and students" safety awareness, and scientific awareness of safety risks. Establish safety access system for teaching laboratories, provide teachers and students entering the laboratory with safety skills and operational practice training. Those who fail to receive obtained relevant safety education or qualified results cannot enter the teaching laboratory. Enrich laboratory safety education content by distributing learning materials, carrying out various lectures, conducting safety knowledge competitions, watching videos and classroom teaching. Safety publicity education should be a required content during daily safety examination. It is necessary to inspect safety accidents responsibility for safety education training. 


\subsection{Establishment and implementation of feasible laboratory safety management system}

Only by starting from system construction, establishing a set of strict, effective, feasible and targeted rules and regulations, and strictly executing in daily laboratory work, guaranteeing the full implementation of management systems, laboratory safety accidents can be effectively avoided. occur. Establish laboratory leadership management system with specially-assigned person in charge. Establish laboratory with dedicated full-time management staff in charge from the school and institute level, continuously sort out management system, clarify management responsibilities, achieve effective laboratory safety management supervision, and promote colleges and universities to establish organizations fully responsible for safety.

\subsection{Establishment and perfection of laboratory emergency plan for laboratory sudden safety accidents}

After combining characteristics of laboratory safety accident occurrence at home and abroad which happen suddenly, it is hard to completely eliminate in daily management. Thus, to effectively deal with various laboratory emergency accidents, it is particularly important to establish emergency plan and mechanism for laboratory accidents. The scientific and reasonable emergency plan and mechanism for laboratory accidents can reduce the number of casualties and property losses after the accident. Formulation of the emergency plan for sudden laboratory safety accidents make it possible to avoid panic caused by the accident, so that related responsible parties involved can take a tense situation calmly, quickly cope with accident and reduce the loss to the minimum.

Currently, Chinese universities have formulated emergency plans for sudden laboratory safety accidents which effectively avoid further personnel and property loss after accidents. However, some deficiencies are found in emergency plans during practical applications, needing further condensing and perfecting in practice. Meanwhile, it is necessary to practice the emergency plan in routine laboratory safety management, and entirely improve laboratory safety management level.

\section{Conclusion and prospect}

Since reform and opening up, with the rapid development of China's higher education, the number and types of experimental teaching are becoming complicated. Laboratory is the most important base for experimental teaching but university laboratory safety accidents occur frequently under the current condition. How to guarantee experimental teaching to be carried out smoothly and steadily in a safe environment is a major task in front of university laboratory safety management researchers and implementers.

University laboratory safety loopholes have attracted the attention of colleges and the society. The laboratory safety management construction system is under continuous development and perfection. In university laboratory safety management, it is necessary to adhere to the principle of prevention and safety first, and establish complete laboratory safety management system from the aspects of improving laboratory safety hardware conditions, improving teachers and students" safety management awareness, and strengthening laboratory safety work systems and prevention mechanism construction, so as to effectively eliminate the occurrence of laboratory accidents and create a harmonious experimental environment for teachers and students.

\section{References}

[1] Chang Jingzhu. Analysis on University Economic Management Laboratory Safety Management Problems, China Education Innovation Herald (Mid-term), 2018(5).

[2] Wen Guanghao, Zhou Qin, Cheng Lei. Strengthen Laboratory Safety Management and Improve Laboratory Management Level, Experimental Technology and Management, 2009, 26(4):153-157.

[3] Huang Kun, Li Yanqi. Analysis of the Current Situation of University Laboratory Safety Management in China and Countermeasures, Laboratory Research and Exploration, 2015, 
34(1):280-283.

[4] Chang Shenghua, Weng Xiuxiu, Hou Fujiang. Analysis and Research on the Current Situation of University Laboratory Safety Management, Experimental Technology and Management, 2016, v.33; No.232(1):229-231.

[5] Lv Changping, Zhou Fengqi, He Xi. The Current Situation and Countermeasures of University Laboratory Safety Management System, Experiment Technology and Management, 2017, 34(2): 242-244.

[6] Huang Kai. Thinking and Practice of Constructing University Laboratory Safety Management System, Experimental Technology and Management, 2016, 33(12):1-4. 